\title{
Effects of Musically-Induced Emotions on Choice Reaction Time Performance
}

\author{
Daniel T. Bishop, Costas I. Karageorghis, \& Noel P. Kinrade \\ Brunel University
}

\begin{abstract}
The main objective of the current study was to examine the impact of musically-induced emotions on athletes' subsequent choice reaction time (CRT) performance. A random sample of 54 tennis players listened to researcher-selected music whose tempo and intensity were modified to yield six different music excerpts (three tempi $\mathrm{x}$ two intensities) before completing a CRT task. Affective responses, heart rate (HR), and RTs for each condition were contrasted with white noise and silence conditions. As predicted, faster music tempi elicited more pleasant and aroused emotional states; and higher music intensity yielded both higher arousal $(p<.001)$ and faster subsequent CRT performance $(p<.001)$. White noise was judged significantly less pleasant than all experimental conditions $(p<.001)$; and silence was significantly less arousing than all but one experimental condition $(p<.001)$. The implications for athletes' use of music as part of a preevent routine when preparing for reactive tasks are discussed.
\end{abstract}

Research into the emotional impact of music listening is burgeoning, most notably to further our understanding of neurophysiological emotion phenomena (e.g., Gosselin, Peretz, Johnsen, \& Adolphs, 2007); and recent developments in digital music products such as the iPod have enabled people to privatize their auditory environment, allowing them to moderate the content and intensity of their emotions. Music is not only ubiquitous and portable, but also regularly employed as an emotion management tool by young athletes (Bishop, Karageorghis, \& Loizou, 2007). Nonetheless, the effects of musically-induced emotions on subsequent sport performance have not been the subject of empirical investigation.

Appraisal theorists (e.g., Lazarus \& Folkman, 1984) posit that emotional responses are brought about by an individual's appraisals of events and situations, wherein the perceiver evaluates the personal significance of an event for his or her wellbeing according to a number of criteria, such as intrinsic pleasantness. Frijda (1987) introduced the concept of action tendencies, which Frijda, Kuipers, and ter Schure (1989) later described as "what links experience and behavior ... a reflection of the actual state of behavioral readiness" (p. 213). Thus, action tendencies are primarily adaptive psychomotor states which enable us to initiate the most

Bishop, Karageorghis, and Kinrade are with the School of Sport and Education, Brunel University, West London, Uxbridge, Middlesex UB8 3PH, England, UK. 


\section{TSP GALLEY PROOF}

appropriate motor response in any given situation; they can be broadly classified as approach- or avoidance-directed, typically culminating in movement toward or away from the emotion-inducing stimulus, respectively (e.g., we are primed for evasive movement in the face of threat).

Appraisal also plays a key role in the musical elicitation of emotions (Scherer $\&$ Zentner, 2001). Musically-elicited emotions are not only incredibly diverse, but can also exhibit high intensity (Gabrielsson, 2001). Indeed, Scherer (2004) noted that music has the power to elicit utilitarian emotions, which promote hormonal, endocrine and autonomic changes; they also initiate appropriate action tendencies-driven by appraisals in the central nervous system. Utilitarian emotions are comparable to primary emotions, which have evolutionary significance for our survival. Scherer suggests that the affective (feeling) component of such emotions reflects concurrent changes in all other components - the result of event appraisal, change in motivational direction and/or levels, and proprioceptive feedback from motor expressions and physiological reactions (cf. Scherer \& Zentner, 2001).

Mood and emotions in sport have attracted considerable research interest (Cerin, 2003; Jones, 2003; Terry, 2004). Athletes are frequently asked to retrospectively recall competitive emotional states, which can lead to misreporting of emotional intensity (Tenenbaum \& Elran, 2003). However, the Affect Grid (Russell, Weiss, \& Mendelsohn, 1989), derived from Russell's (1980) circumplex model of affect, is a single-item measure which allows for more expedient in vivo collection of affective data. The original circumplex comprises two bipolar perpendicular dimensions-perceived activation (arousal) and affective valence (pleasure-displeasure)-yielding four quadrants; proponents of the model contend that all experienced emotions can be located at some point within one of the four quadrants. The Affect Grid has since been used to differentiate affective states in sporting contexts (Edmonds, Mann, Tenenbaum, \& Janelle, 2006; Hardy, Hall, \& Alexander, 2001).

The two orthogonal dimensions of valence and arousal appear to be reliable predictors of emotional experiences with music (North \& Hargreaves, 1997; Ritossa \& Rickard, 2004) - a finding which has been mirrored in cardiorespiratory data. Nyklícek, Thayer, and Van Doornen (1997) investigated the capacity of cardiorespiratory variables to differentiate discrete emotions which had been semantically defined beforehand. Twenty-six participants aged 18-26 yrs listened to 12 music excerpts depicting four emotions (happy, serene, sad, and agitated), as well as to a white noise condition, during continuous acquisition of cardiorespiratory data. Their results supported the notion that cardiorespiratory discrimination of discrete emotions can be located somewhere along a limited number of higherorder dimensions, such as arousal and valence.

Russell's (1980) circumplex model also has heuristic value, because it suggests a clear structure for the effects that emotions can have on behavior (Larsen \& Diener, 1992). J.F. Thayer and Faith (2001) suggested that valence represents the evaluative outcome necessary to initiate an approach or withdrawal response, while arousal reflects the resource investment in the action tendency (Frijda, 1987). However, the speed of the motor output may depend not only on the arousal value of the stimulus, but also on its survival value: Coombes, Cauraugh, and Janelle (2007) noted that the premotor reaction time component of participants' arm extension movement (characterized by the authors as a withdrawal/avoidance 
response) was quicker in response to threat-related stimuli than similarly arousing stimuli without such clear survival implications. However, it should be noted that arm extension is not inherently a manifestation of an avoidance tendency; an action's affective-motivational significance is very much dependent on the context in which it occurs (Markman \& Brendl, 2005). Nonetheless, generalized forward locomotion is considered to be a fundamental characteristic of exploratory/ approach-type behavior (Panksepp, 1998).

According to Lang (2000), behavioral responses can be conditioned to otherwise innocuous secondary cues such as lights, tones or music. Lang highlighted our relatively poor understanding of emotional reactions' impact on athletic performance, despite widespread agreement as to their unquestionable influence; he also noted the widespread absence of an overt behavioral action measure when assessing the efficacy of emotion manipulation in the laboratory. Therefore, a CRT measure necessitating forward locomotion and arm extension may represent an ecologically valid means by which to assess the impact of emotion manipulation on subsequent reactive performance in tennis: The first serve takes approximately half a second to reach the opposing baseline; the returning player must make the appropriate adjustments within this brief timeframe to be optimally positioned to strike the ball, and this typically requires forward locomotion and arm extension.

When considering the impact of music listening on affective and behavioral responses, one must consider both intrinsic (e.g., acoustical properties) and extrinsic sources (e.g., classically conditioned associations) of emotion. Although extrinsic sources are arguably the more potent inducers of emotion (Rickard, 2004), intrinsic sources have the advantage of being readily manipulable. One such intrinsic source of emotion in music is its tempo; faster tempi are associated with more positive valence. Webster and Weir (2005) examined the interaction of tempo (72, 108, and 144 beats per minute), texture (harmonized vs. nonharmonized) and mode (major vs. minor) on listeners' affective responses. They found that music played in major modes, at faster tempi, with harmonized melodies, elicited happier responses in the listener; this emotional response has also been shown to emerge at an early developmental stage (Dalla Bella, Peretz, Rousseau, \& Gosselin, 2001), suggesting something primal about the relationship between valence and tempo.

Music intensity (volume) appears to strongly moderate the arousal component of emotional responses to music in tennis players (Bishop et al., 2007); and higher musically-induced arousal may also yield enhanced cognitive processes, as reflected in electrophysiological data: Schmidt and Trainor (2001) used EEG to examine cortical responses to musical excerpts preselected for their collective representation of different affective valences (i.e., positive and negative) and intensities (i.e., intense and calm). When contrasted with happy and sad music, overall frontal activity was more pronounced in the more intense musically-elicited emotions of fear and joy; these latter emotions have clear avoidance and approach action tendencies associated with them, respectively. Positive and negative emotions with such action tendencies are present in facilitative emotional profiles in sport (Cerin, 2003); and activation of right prefrontal cortex appears to be associated with the determination of service direction in tennis (Wright \& Jackson, 2007). 
Musical accompaniment has been shown to improve motor reaction time (Bassagaoglu, Kalkan, \& Sari, 2004). In addition, music intensity designated as comfortable may be more conducive to superior performance when played concurrently with the task. Turner, Fernandez, and Nelson (1996) asked participants to perform a motor-reaction task in the presence of popular music played at four different intensities (No music, $60 \mathrm{dBA}, 70 \mathrm{dBA}$, and $80 \mathrm{dBA}$ ). Participants performed significantly faster in the $70 \mathrm{dBA}$ condition, wherein the music intensity was closest to their comfort levels. Neurophysiological evidence indicates that fast tempo music played during performance of a demanding visual selective attention task leads to enhanced stimulus evaluation, reflected in reduced latency of visual attention event-related potentials (Amezcua, Guevara, \& Ramos-Loyo, 2005). Prestimulus EEG activity has also been shown to correlate with performance on an acoustic choice reaction-time task (Winterer, Adams, Jones, \& Knutson, 2002); and preparatory arousal "appears to be the most effective strategy" for dynamic tasks (Tod, Iredale, \& Gill, 2003). This suggests an avenue for employing preperformance arousal management strategies such as listening to appropriately selected music. However, the residual effects of musically-induced emotions on performance are yet to be elucidated.

In light of evidence for the contribution of intensity and tempo to emotional responses to music in tennis (Bishop et al., 2007), the role of tempo in enhancing attentional processes (Amezcua et al., 2005), and the mediatory effects of motivational direction on reactive performance (Coombes et al., 2007), the aim of the current study was to examine the interrelationships between changes to the tempo and intensity of music heard, resultant affective responses, and subsequent CRT task performance. It was predicted that listening to fast tempo, high intensity music would induce a more highly aroused and positively valenced affective state than (a) comparatively slower and quieter music, (b) white noise, and (c) silence; and that this emotional state would additionally be manifested in higher HRs and faster performance on a CRT task requiring approach-type behaviors.

\section{Method}

\section{Participants}

An international tennis academy in southwest London, England, UK was chosen as a suitable site for data collection. Participants volunteered, but were incentivized to take part with prizes (for first, second, and third places) as a part of an open-to-all competition within the academy. Pilot testing revealed a required sample size of 46, based on an alpha level of .05 and a desired power of .9. Fiftyfour participants, comprising 33 males and 21 females, were recruited to guard against participant dropout and deleted cases owing to multivariate outliers. Participants' ages ranged from 13 to 22 years $(M=17.7, S D=2.1$ years $)$. Thirty-six participants described their ethnicity as "White UK"; the remaining 18 participants comprised 13 other ethnicities. Participants' competitive experience ranged from 24 to 156 months $(M=84.8, S D=34.1$ months $)$, and their abilities ranged from county level to world ranked; Lawn Tennis Association (LTA) ratings ranged from 1.1 to 9.2. All participants were right-handed. 


\section{Equipment and Materials}

Auditory Stimuli. The music track selected was Deepest Blue by the artist Deepest Blue, which was sold under the Data record label (catalog no. 55CDS). This track was selected because it satisfied a number of criteria: Its UK Chart (Official UK Charts Company, London, UK) Top 40 entry date of second August 2003 (during the summer vacation period for all mainstream UK schools), its peak position of number seven in the charts, and its eight weeks spent in the Top 40 collectively meant that it was likely to be familiar to all participants; a quality which appears to be related to the degree of cultural exposure (North \& Hargreaves, 1995).

Three music tempi were created using a digital music player's Time Scaling feature, to yield three recorded excerpts played at $99 \mathrm{bpm}$ (slow tempo), $129 \mathrm{bpm}$ (normal tempo), and $161 \mathrm{bpm}$ (fast tempo). All excerpts were cropped to $90 \mathrm{~s}$ in length, enabling participants to hear a portion of the verse and chorus. The intensity of each excerpt was increased to produce three new ones. The final moderate intensity stimuli were played at $55 \mathrm{dBA}$, whereas loud intensity stimuli were played at $75 \mathrm{dBA}$. Two digital white noise excerpts $90 \mathrm{~s}$ in duration and a $90 \mathrm{~s}$ period of silence were also recorded, so that nine auditory stimuli conditions were created in total: Six music excerpts (three tempi $\times$ two intensities), two white noise excerpts (two intensities), and one silence period. Auditory stimuli were presented in a randomized order via earbud-type earphones (Creative EP-630, Creative Laboratories, Inc., Milpitas, California).

Affective and HR Responses. Participants recorded their affective responses to music by marking a cross on the Affect Grid (Russell et al., 1989), and elaborated on this by circling affective descriptors (mirroring Russell's [1980] original list) which they deemed to most closely represent their affective state. Participants' HR was monitored continuously throughout the study using a chest strap-type HR monitor with infrared PC communication capabilities (Polar S610i; Polar Electro Oy, Kempele, Finland), at a 5 s sampling rate. The researcher recorded (a) the start time for recording HR data and (b) the times at which the participant commenced and ceased the CRT task, to facilitate subsequent analysis of HR data.

CRT Task. The study protocol was created and executed, and reaction time (RT) data recorded, using experiment generator software (E-Prime v.1.1.4.1; Psychology Software Tools, Inc., Pittsburgh, Pennsylvania, US). RT was defined as the time in ms from stimulus onset to a recorded response. Visual stimuli were presented using a computer projector (Hitachi CP-RS56; Hitachi Ltd., Tokyo, Japan) onto a white wall; the total projected image measured $1.29 \mathrm{~m}$ in width by $1.03 \mathrm{~m}$ in height, at a distance of approximately $2.6 \mathrm{~m}$ from the participant. The stimulus to which participants responded-an image of a ball-was projected onto the retina at a diameter of $0.99 \mathrm{~mm}$, which corresponds to a tennis ball at a distance of $1.72 \mathrm{~m}$ away from the returning player (after the ball has landed, when the player is on or behind the baseline). The tennis court image backdrop represented the returning player's viewpoint when returning from the left-hand side (commonly referred to as the "Ad side") of the court.

The ball stimulus could appear in one of three locations: Left (representing a serve out wide), center (representing a serve to the body), or right (representing a 
serve along the center service line). Participants stood behind a Table $0.75 \mathrm{~m}$ high on which three circular buttons measuring $0.09 \mathrm{~m}$ in diameter were mounted; each button corresponded to one of the three ball stimuli. Participants responded to visual stimuli with their dominant hand (i.e., the one used to hold the racket). Participants' starting position was not specified: The counterbalanced randomized repeated-measures design of the study meant that no performance advantage was conferred by adopting any one position; participants ultimately responded to each of the three locations an equal number of times. Figure 1 depicts the experimental setup.

\section{Procedure}

Following institutional ethics approval, a randomized repeated-measures design was employed wherein participants completed all nine conditions. Each participant was informed of what would be expected of them, and were invited to voice any concerns. After one practice block of the CRT task, the participant sat in the chair wearing the earphones. A projected image displayed relaxation instructions (available from first author) for $45 \mathrm{~s}$, immediately followed by a $90 \mathrm{~s}$ listening block, accompanied by a blank screen. Midway through the listening block, the first author prompted the participant to record his or her affective response.

After the participant had listened to the excerpt and recorded his or her responses, a third screen of $20 \mathrm{~s}$ duration appeared which prompted the participant to stand up from the chair and prepare for CRT task performance. The participant then responded with his or her dominant hand as quickly as possible to each of 24

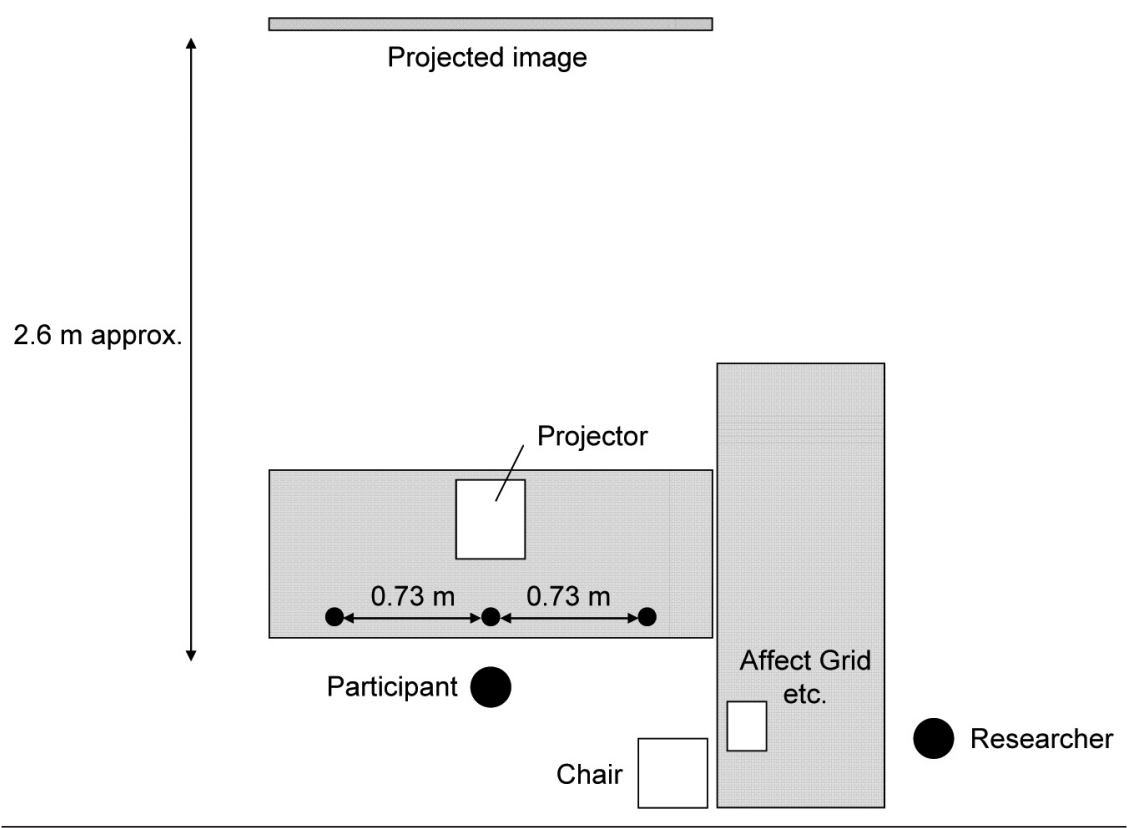

Figure 1 - Experimental setup. 
stimuli ( $8 \times 3$ ball locations); all trials were presented in a new counterbalanced randomized order in each block. A screen depicting a blank court preceded each stimulus, for $1 \mathrm{~s}$ duration. Experimental stimuli moved on once a participant's response had been registered, or after $1 \mathrm{~s}$ in the event of a nonresponse. After disappearance of the last of the 24 stimuli, the relaxation instructions reappeared. This procedure was repeated a further eight times.

Manipulation Check. The first author asked the following question immediately after completion of data collection: "Did the slowed-down and sped-up versions of the track appear significantly different to the original version in any way other than the speed?" In addition, each participant was asked, "Was the original version of the track you heard familiar to you prior to participating in this study?" to gauge the success of the selection strategy; familiarity moderates preference for musical stimuli (Berlyne, 1971), and extreme ratings for valence might have confounded results.

\section{Qualitative Data Analysis}

Mean Affect Grid (Russell et al., 1989) ratings of each of the 28 descriptors were used to plot the position of each in two-dimensional space-pleasantness (abscissa) $\times$ arousal (ordinate), as per Russell's (1980) original circumplex model.

\section{Quantitative Data Analysis}

Reaction time data were imported into a software package which enables grouping of participants' individual E-Prime data (E-DataAid v.1.1.4.1; Psychology Software Tools, Inc., Pittsburgh, Pennsylvania, US). Participants' error rates during response to experimental stimuli were negligible $(0.29 \%)$, and were automatically excluded from the analysis using E-DataAid's Filter option. HR data were uploaded to HR monitor data management software (Polar Precision Performance SW; Polar Electro Oy, Kempele, Finland). Missing data points were accounted for by averaging of adjacent values (see Tabachnick \& Fidell, 2007). Average HR data were calculated for each CRT task period.

CRT data, HR data, and Affect Grid (Russell et al., 1989) data were transferred to a workbook in spreadsheet software (Microsoft Excel 2002; Microsoft Corporation, Fargo, ND, US), before transfer to statistical analysis software (SPSS 15.0 for Windows; SPSS Inc., Chicago, IL, US). A one-way repeated measures (RM) MANCOVA was used to analyze the reaction time, arousal, pleasantness, and HR data; LTA rating was used as a between-subjects mean-centered covariate (see Delaney \& Maxwell, 1981), because level of expertise may mediate performance under pressure (Beilock \& Carr, 2001). Wilks's Lambda was chosen as the omnibus statistic, owing to the relatively large sample size, equal $N$ values, and independence of observations (Tabachnick \& Fidell, 2007, p. 348). Follow-up $F$-tests and Bonferroni-adjusted pairwise comparisons were computed to examine where differences lay. 


\section{Results}

\section{Qualitative Data Analysis}

Figure 2 shows that descriptors are arranged in a structure which corresponds closely to that of Russell's (1980) original conceptualization of the affective circumplex model.

\section{Quantitative Data Analysis}

Intercorrelations of Dependent Variables (DVs). Scores on the covariate, LTA rating, did not correlate with any DV scores. All remaining DV intercorrelations were nonsignificant, $p>.05$, except for that between arousal score and RT, $r(486)$ $=-.14, p<.01$. Notably, arousal scores and pleasantness score exhibited a nearzero correlation, $r(482)=-.07, p>.05$, underlining the orthogonality of pleasantness and arousal dimensions (cf. Russell, 1980).

Main Effect of Condition. LTA rating had no significant effect, nor did it interact significantly with condition, $p>.05$. There was a main effect of condition, Wilks's Lambda (.02), $F(32,21)=27.90, \eta_{\mathrm{p}}{ }^{2}=.98, p<.001$. Mauchly's Test of Sphericity showed that the data for all dependent variables had violated the assumption of sphericity. Therefore, follow-up $F$ tests with Greenhouse-Geisser adjustment were used. Descriptive and univariate statistics (see Table 1) indicate that there were differences between conditions for three of the four dependent variables-reaction time, arousal score, and pleasantness score.

Follow-up pairwise comparisons indicated only one significant $(p<.05)$ difference in CRT performance (see Table 1). Specifically, reaction time subsequent to listening to Fast-Loud Music was lower than that after listening to Fast-Moderate Music. Fast-Loud Music also elicited significantly $(p<.001)$ higher arousal ratings than Normal-Moderate Music, Slow-Loud Music, Slow-Moderate Music, Moderate White Noise, and Silence. Silence elicited significantly $(p<.001)$ lower arousal ratings than all conditions, with the exception of Slow-Moderate Music ( $p$ $>$.05). Loud White Noise and Moderate White Noise were rated as significantly $(p<.001)$ less pleasant than all other conditions. Mean arousal scores, pleasantness scores, and reaction times for all conditions are depicted in Figure 3.

Main Effects of Tempo and Intensity (Music Conditions Only). There was no main or interaction effect of the covariate, $p>.05$. A second one-way RM MANCOVA was used to analyze the main effects of tempo and intensity for the music conditions only. There was a significant Tempo $\times$ Intensity interaction, Wilks's Lambda $(.71), F(8,45)=2.36, \eta_{\mathrm{p}}{ }^{2}=.30, p<.05$. There were significant main effects of tempo, Wilks's Lambda $(.26), F(8,45)=16.18, \eta_{\mathrm{p}}^{2}=.74, p<.001$; and intensity, Wilks's Lambda $(.58), F(4,49)=9.06, \eta_{\mathrm{p}}{ }^{2}=.43, p<.001$. A series of one-way RM ANCOVAs was conducted to examine differences between levels of the independent variables for each of the four dependent variables. Mauchly's Test of Sphericity indicated that the data satisfied the assumption of sphericity, with the exception of the arousal score data for tempo, Mauchly's $W(2)=.86, \chi^{2}$ $=7.98, p<.05$; and reaction time data for the Tempo $\times$ Intensity interaction, 
TSP GALLEY PROOF

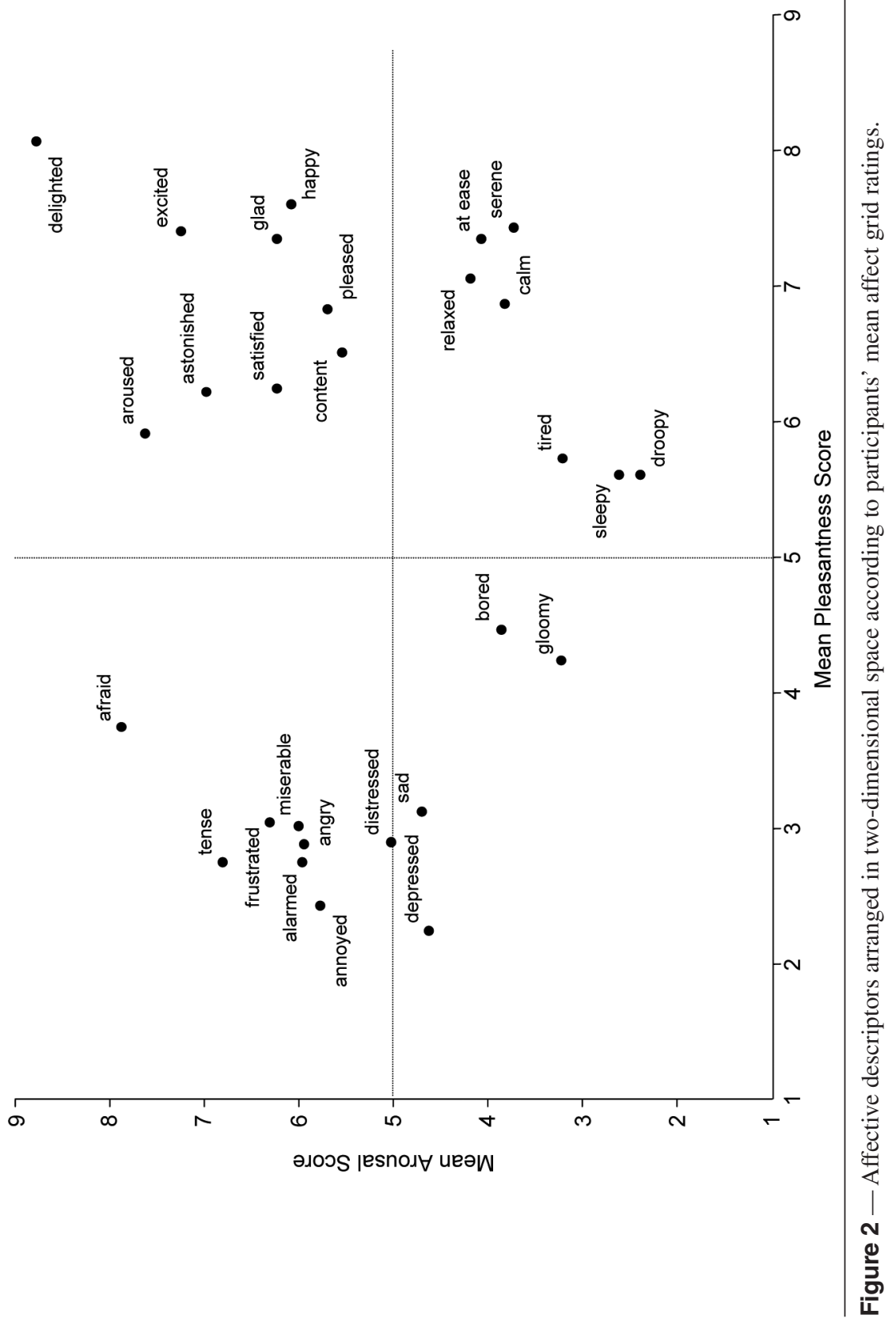


Table 1 Descriptive Statistics and F Tests for all Dependent Variables (DVs)

\begin{tabular}{|c|c|c|c|}
\hline DV & Condition & $M$ & $S D$ \\
\hline \multirow{9}{*}{$\begin{array}{l}\text { Reaction time } \\
(\mathrm{ms})\end{array}$} & Fast-loud music & 492.97 & 58.47 \\
\hline & Fast-moderate music & 511.25 & 58.33 \\
\hline & Normal-loud music & 505.22 & 68.35 \\
\hline & Normal-moderate music & 507.02 & 52.34 \\
\hline & Slow-loud music & 487.08 & 65.04 \\
\hline & Slow-moderate music & 501.71 & 54.82 \\
\hline & Loud white noise & 498.02 & 59.31 \\
\hline & Moderate white noise & 510.50 & 49.34 \\
\hline & Silence & 505.91 & 63.01 \\
\hline \multicolumn{4}{|c|}{$F(6.19,322.10)=2.33, \eta_{\mathrm{p}}^{2}=.04, p<.05$} \\
\hline \multirow{9}{*}{$\begin{array}{l}\text { Arousal } \\
\text { score }\end{array}$} & Fast-loud music & 6.57 & 2.31 \\
\hline & Fast-moderate music & 6.44 & 1.83 \\
\hline & Normal-loud music & 5.56 & 2.20 \\
\hline & Normal-moderate music & 5.09 & 2.02 \\
\hline & Slow-loud music & 4.57 & 2.34 \\
\hline & Slow-moderate music & 3.26 & 1.94 \\
\hline & Loud white noise & 6.11 & 2.03 \\
\hline & Moderate white noise & 4.74 & 2.11 \\
\hline & Silence & 2.94 & 1.92 \\
\hline \multicolumn{4}{|c|}{$F(4.77,248.21)=23.91, \eta_{p}^{2}=.32, p<.001$} \\
\hline \multirow{9}{*}{$\begin{array}{l}\text { Pleasantness } \\
\text { score }\end{array}$} & Fast-loud music & 6.28 & 2.03 \\
\hline & Fast-moderate music & 6.89 & 1.83 \\
\hline & Normal-loud music & 6.28 & 2.14 \\
\hline & Normal-moderate music & 6.46 & 1.89 \\
\hline & Slow-loud music & 5.43 & 2.51 \\
\hline & Slow-moderate music & 5.85 & 2.09 \\
\hline & Loud white noise & 2.91 & 2.62 \\
\hline & Moderate white noise & 3.52 & 2.46 \\
\hline & Silence & 7.13 & 1.95 \\
\hline \multicolumn{4}{|c|}{$F(5.49,285.57)=29.06, \eta_{p}^{2}=.36, p<.001$} \\
\hline \multirow{9}{*}{$\begin{array}{l}\text { HR } \\
\text { (bpm) }\end{array}$} & Fast-loud music & 86.56 & 12.95 \\
\hline & Fast-moderate music & 87.03 & 12.65 \\
\hline & Normal-loud music & 88.50 & 11.68 \\
\hline & Normal-moderate music & 86.06 & 12.09 \\
\hline & Slow-loud music & 87.61 & 12.25 \\
\hline & Slow-moderate music & 87.88 & 12.69 \\
\hline & Loud white noise & 87.07 & 11.04 \\
\hline & Moderate white noise & 86.90 & 12.52 \\
\hline & Silence & 87.01 & 12.57 \\
\hline
\end{tabular}

Note. $F(6.10,317.43)=1.35, \eta_{\mathrm{p}}{ }^{2}=.03, p>.05$ 
Reaction Time (s)

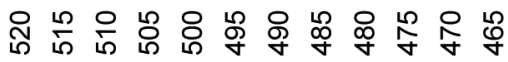

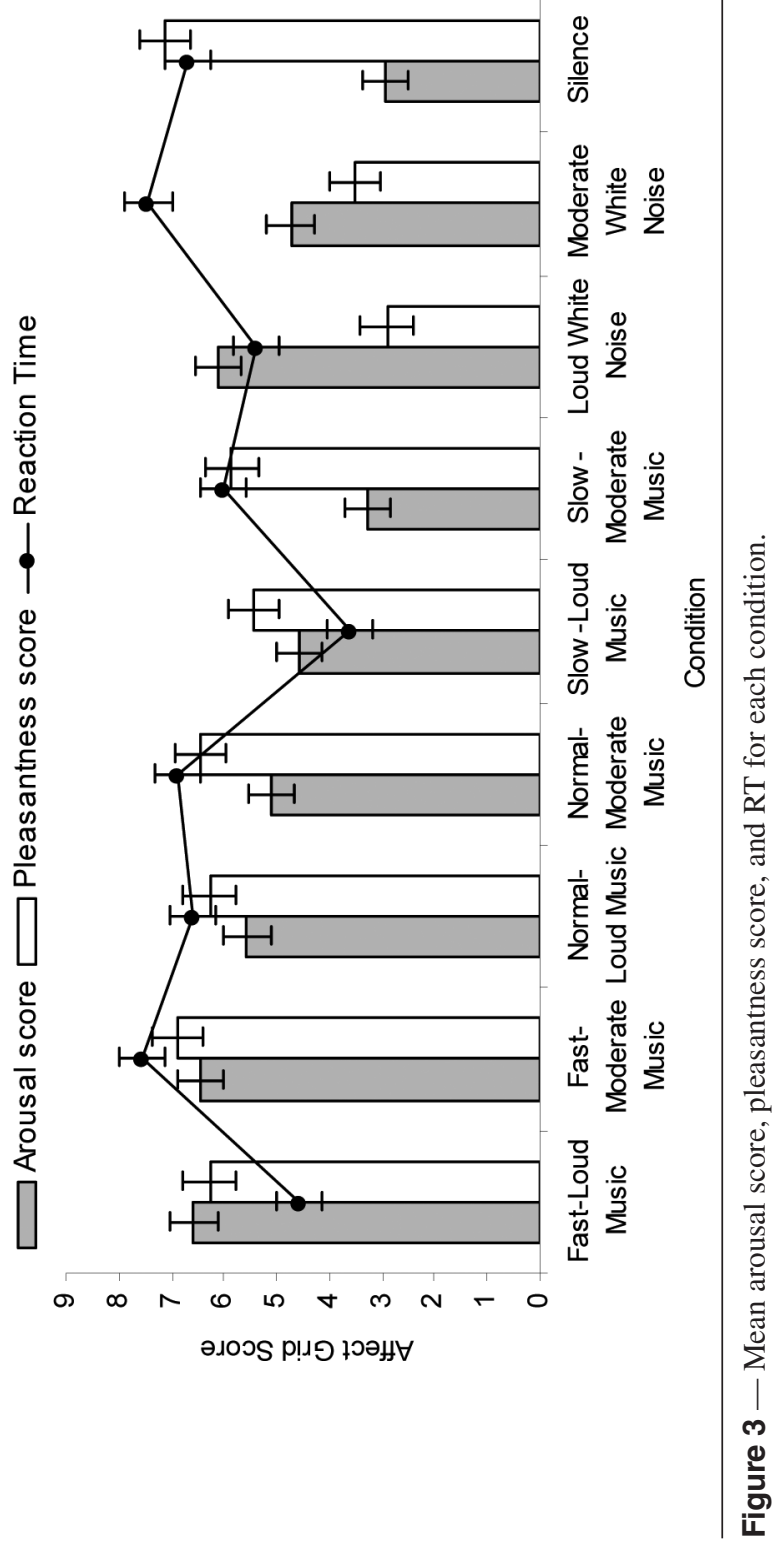


Mauchly's $W(2)=.85, \chi^{2}=8.24, p<.05$. Therefore, Greenhouse-Geisser adjustment was applied to the corresponding $F$ tests.

There was no significant Tempo $\times$ Intensity interaction $(p>.05)$. However, there was a significant main effect of tempo for arousal score, $F(1.75,90.84)=$ $40.21, \eta_{\mathrm{p}}{ }^{2}=.44, p<.001$, and pleasantness score, $F(1.91,99.38)=10.19, \eta_{\mathrm{p}}{ }^{2}=$ $.16, p<.001$. There was also a significant main effect of intensity, for reaction time, $F(1,52)=7.96, \eta_{\mathrm{p}}{ }^{2}=.13, p<.01$, and arousal score, $F(1,52)=15.71, \eta_{\mathrm{p}}{ }^{2}$ $=.23, p<.001$. Follow-up pairwise comparisons (see Table 2 ) indicated that fast tempo music elicited significantly $(p<.001)$ higher arousal scores than did normal tempo music, which yielded significantly $(p<.001)$ higher subjective arousal than did slow tempo music. Fast tempo music engendered significantly $(p<.01)$ more positive valence than did slow tempo music; and normal tempo music produced significantly $(p<.01)$ more positive valence than did slow tempo music. Loud intensity music elicited significantly $(p<.01)$ shorter reaction times-and significantly $(p<.001)$ higher subjective arousal—-than did moderate intensity music.

\section{Manipulation Check}

All participants indicated that, aside from noticing that the slowed-down and speeded-up versions were slower and faster respectively, there were no other perceptible differences. All participants recalled that they had previously heard the original version of the track.

\section{Discussion and Conclusions}

The primary aim of this study was to examine the affective and behavioral consequences of listening to six varied musical excerpts ( 3 tempi $\times 2$ intensities) of a single researcher-selected track, and to contrast this with the consequences of listening to (a) white noise played at two intensities and (b) a period of silence. There was only partial support for the experimental hypotheses. Chiefly, music listening per se did not improve subsequent CRT performance relative to silence or white noise, irrespective of tempo or intensity; but music played at a fast tempo and high intensity did yield shorter RTs in a subsequent CRT task than did fast, moderate intensity music. White noise conditions were judged significantly less pleasant than all other conditions, and the size of this effect was large $\left(\eta_{p}^{2}=.36\right)$. Silence was less arousing than all but slow, moderate intensity music. There was no effect of condition on participants' HRs. On examination of music conditions only, faster music tempi induced higher valence and arousal; loud music elicited heightened arousal and shorter RTs. LTA rating, a measure of playing ability, did not mediate any of the aforementioned relationships.

The finding that music played at loud intensity yielded higher arousal and faster CRT performance than that played at a moderate intensity may reflect higher energetic investment in the resultant action tendency (J.F. Thayer \& Faith, 2001). This is also consistent with the presence of increased frontal lobe activity before superior CRT performance (Winterer et al., 2002) and during experience of highintensity emotions (Schmidt \& Trainor, 2001). In accordance with appraisal theories of emotion (e.g., Scherer, 2004), it was hypothesized that music appraised as highly arousing would elicit approach-type action tendencies/exploratory behav- 


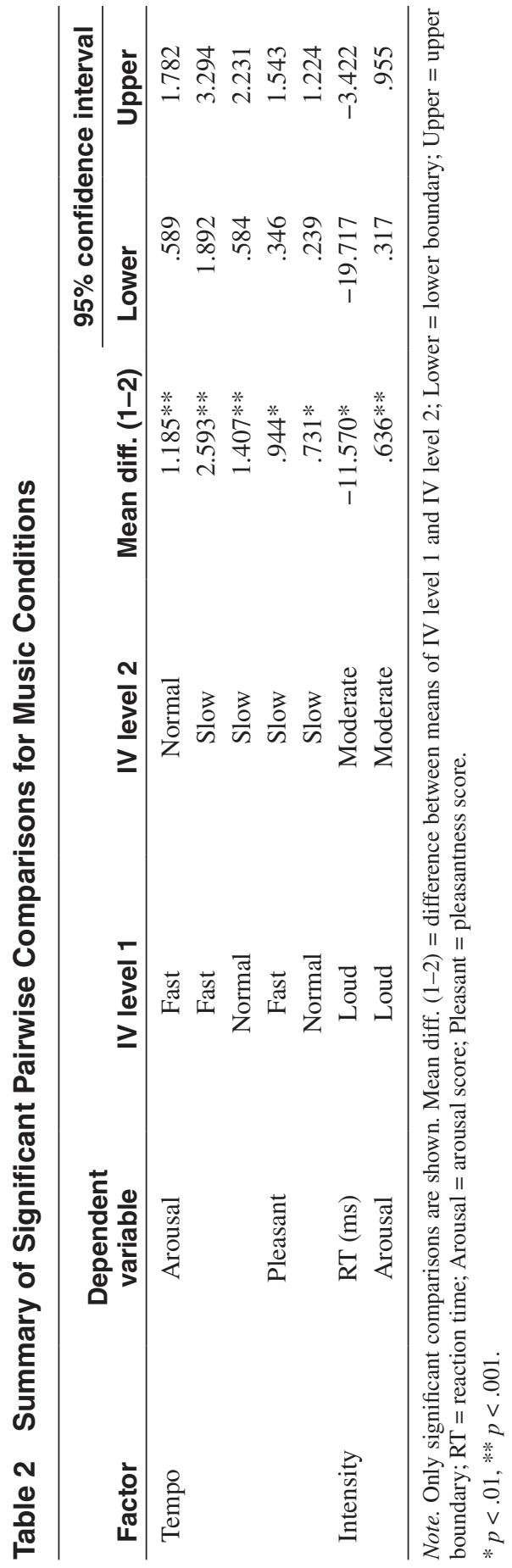


ior-a defining feature of which is forward locomotion (Panksepp, 1998); such motion was required to perform the present CRT task. However, the correlation between arousal and performance was very low $(r[486]=-.14)$. In future studies, a physiological measure of arousal may afford more definitive conclusions on the music-arousal-performance relationship.

Present data indicate that listening to music is preferable to general noise of the same intensity (white noise in the current study) if a positive emotional state is desired. Noise is an inevitable part of the competitive tennis environment when preparing for a match, and silence-which was judged similarly pleasant to music - is all-but impossible to attain. However, MP3 technology enables the athlete to create a privatized auditory environment in which he or she can engender a positive emotional state; indeed, music listening enables a range of young tennis players' emotion management needs to be fulfilled (Bishop et al., 2007). Given the apparent contributions of tempo and intensity to participants' arousal, increasing one or both of these variables may be a suitable means by which athletes can psych-up to improve muscular force output (cf. Tod et al., 2003). Examination of Figure 2 reveals that descriptors associated with high valence and high arousal (mean scores $\geq 6$ ) were astonished, delighted, excited, glad, and satisfied. Some of these descriptors mirror Scherer's (2004) utilitarian emotions, and may be appropriate target affective states for athletes looking to concomitantly increase emotional valence and arousal.

The present findings add to extant research which indicates that tempo is a strong determinant of affective responses to music (Dalla Bella et al., 2001; Webster \& Weir, 2005): Music excerpts played at both fast and normal tempi were considered significantly more pleasant than music played at a slow tempo; and the effect was strong $\left(\eta_{\mathrm{p}}{ }^{2}=.16\right)$. There was also a very strong effect on arousal $\left(\eta_{\mathrm{p}}{ }^{2}=\right.$ .43). One plausible explanation is that faster tempi are appraised as being representative of high energetic arousal (R. E. Thayer, 1989), a desirable state for all living organisms which indicates health and vitality; its polar opposite-low energetic arousal-is indicative of undesirable psychophysiological states such as depression and illness. However, faster tempi did not lead to shorter RTs, despite the fact that they elicited greater subjective arousal. Nonetheless, positive affective states engender cognitive flexibility, mediated by dopaminergic pathways (Lang, 2000)—which also promote increased motor activity. Athletes may be able to use available mp3 technology to speed up their favorite track to enhance affect, although further research is required to clarify the impact of such manipulations on subsequent performance.

Despite the nonsignificant effect of music listening itself on CRT performance, the contributions of fast tempo and louder music intensity to higher arousal-irrespective of valence-are potentially of significance: music that is considered both highly unpleasant and potentially dangerous (i.e., highly disliked fast loud music) may lead to an avoidance-type action tendency. Conversely, highly liked and arousing music may yield approach action tendencies. Nonetheless, this kind of auditory stimulus may elicit approach or exploratory behavior, which includes increased receptiveness to the visual environment (cf. Amezcua et al., 2005), and forward locomotion (Panksepp, 1998); both responses could viably produce faster CRT performance-either in isolation or conjointly. Therefore, lis- 
tening to fast loud music may be a suitable preperformance strategy for tennis players - but this notion requires further exploration.

The finding that loud intensity (75 dBA) music yielded significantly greater arousal and accompanying superior CRT performance than did moderate intensity (55 dBA) music is comparable to Turner et al.'s (1996) findings, wherein music played at $70 \mathrm{dBA}$ was the most conducive to simple RT performance when played concurrently. However, Turner et al. designated this music intensity as comfortable, which serves to highlight the idiosyncratic nature of individuals' responses to music. The impacts of level of expertise and individual differences (e.g., introversion/extraversion traits) on participants' emotional responses to music were not accounted for in the current study, despite evidence to indicate that they may be mediated by the latter, at least (Crust \& Clough, 2006; Kallinen \& Ravaja, 2004). In addition, extrinsic sources of emotion in music (e.g., extramusical associations) are potent determinants of emotional content (Rickard, 2004); more so than intrinsic sources. Therefore, to build on the present data in future studies, it may be prudent to not only use self-selected music, but also to control for potentially mediating effects of participants' personality type and level of expertise.

One notable omission in the current study was the absence of a truly negatively valenced music condition, which meant that the impact of valence on participants' emotional responses could not be adequately assessed. Such conditions are commonplace in the neurophysiological study of musically-induced emotions, although they are typically jumbled concatenations of an otherwise agreeable track (e.g., Menon \& Levitin, 2005). The unanimous familiarity of the track might also have affected participants' responses to the modified versions; familiar stimuli are typically perceived as more preferable, for example (Berlyne, 1971). However, listening to highly disliked music is not a commonplace activity; moreover, orientation away from disharmonious musical stimuli is an innate human response (Zentner \& Kagan, 1996). Therefore, the present design is perhaps more ecologically valid than if such concatenated versions had been included.

HR data in the current study were not informative. The $5 \mathrm{~s}$ sampling rate was unsuitable for detecting subtle emotion-specific cardiac responses, such as that afforded by heart rate variability (HRV) measures used in other music emotion research (e.g., Nyklícek et al., 1997). HR might also have been affected by the competition scenario; similar conditions have been successfully employed to induce pressure in choking research (Beilock \& Carr, 2001). The absence of a standardized baseline period to allow HR recovery from immediately preceding CRT task performance might also have confounded the data obtained. Therefore, future studies should (a) seek to use HRV as a dependent measure in conjunction with affective and behavioral data, to ascertain the degree to which the sympathetic and parasympathetic nervous systems are involved in mediating the affective and behavioral changes witnessed in the current study; and (b) provide sufficient recovery between blocks of trials so as to allow HR values to return to baseline.

Neurophysiological research of emotional responses to music is still very much in its early stages. However, a potentially fruitful next step in the research process would be the acquisition of neurophysiological data (e.g., using functional magnetic resonance imaging [fMRI]), to develop a better understanding of the neural mechanisms underlying the affective and behavioral changes observed 
herein; this would also complement fMRI data relating to the perception of the serve in tennis (Wright \& Jackson, 2007). It is not clear whether observed differences in performance resulted from improved perception, more efficient or forceful movement patterns (cf. Tod et al., 2003), or a combination of these. An exploratory whole-brain analysis of activations during music listening and subsequent CRT task performance will inform the use of music in sporting contexts to a greater extent.

\section{Summary}

In reply to Lang's (2000) earlier call for an explicit emotion-behavior link in sport research, we measured participants' CRT task performance subsequent to manipulation of their emotional state via music listening. Present data show that participants' affective state changed as a result of changes to the tempo and intensity of a selected track. However, there was no clear advantage of listening to music over white noise or silence, in terms of subsequent CRT performance. Nonetheless, increased arousal and positive emotions have both been shown to accompany superior sports performance (Cerin, 2003; Jones, 2003; Tod, 2003). Music listening may be an appropriate and acceptable strategy for athletes wishing to engender a positive and aroused emotional state; fast tempo and loud intensity may maximize the arousal component of the emotional response. The present data do not permit firm conclusions about a musically-induced emotion-behavior link. Further controlled investigation using participant-selected music is required to elucidate the influence of musically-induced emotions on reactive performance.

\section{References}

Amezcua, C., Guevara, M.A., \& Ramos-Loyo, J. (2005). Effects of musical tempi on visual attention ERPs. The International Journal of Neuroscience, 115, 193-206.

Bassagaoglu, I., Kalkan, M.T., \& Sari, N. (2004). The physiological and psychological effects of classical music and pop music on female high school students. Yeni Symposium: A Journal of Psychiatry, Neurology and Behavioral Sciences, 42, 82-90.

Beilock, S.L., \& Carr, T.H. (2001). On the fragility of skilled performance: What governs choking under pressure? European Journal of Social Psychology, 130, 701-725.

Berlyne, D.E. (1971). Aesthetics and psychobiology. New York: Appleton-Century-Crofts.

Bishop, D.T., Karageorghis, C.I., \& Loizou, G. (2007). A grounded theory of young tennis players' use of music to manipulate emotional state. Journal of Sport \& Exercise Psychology, 29, 584-607.

Cerin, E. (2003). Anxiety versus fundamental emotions as predictors of perceived functionality of pre-competitive emotional states, threat, and challenge in individual sports. Journal of Applied Sport Psychology, 15, 223-238.

Coombes, S.A., Cauraugh, J.H., \& Janelle, C.M. (2007). Dissociating motivational direction and affective valence: Discrete emotions alter central motor processes. Psychological Science, 18, 938-942.

Crust, L., \& Clough, P.J. (2006). The influence of rhythm and personality in the endurance response to motivational asynchronous music. Journal of Sports Sciences, 24, 187-195.

Dalla Bella, S., Peretz, I., Rousseau, L., \& Gosselin, N. (2001). A developmental study of the affective value of tempo and mode in music. Cognition, 80, B1-B10. 


\section{TSP GALLEY PROOF}

Delaney, H.D., \& Maxwell, S.E. (1981). On using analysis of covariance in repeated measures designs. Multivariate Behavioral Research, 16, 105-123.

Edmonds, W.A., Mann, D.T.Y., Tenenbaum, G., \& Janelle, C.M. (2006). Analysis of affectrelated performance zones: An idiographic method using physiological and introspective data. The Sport Psychologist, 20, 40-57.

Frijda, N.H. (1987). Emotion, cognitive structure, and action tendency. Cognition and Emotion, 1, 115-143.

Frijda, N.H., Kuipers, P., \& ter Schure, E. (1989). Relations among emotion, appraisal, and emotion action readiness. Journal of Personality and Social Psychology, 57, 212-228.

Gabrielsson, A. (2001). Emotions in strong experiences with music. In P. Juslin \& J.A. Sloboda (Eds.), Music and emotion: Theory and research (pp. 431-449). Oxford, UK: Oxford University Press.

Gosselin, N., Peretz, I., Johnsen, E.L., \& Adolphs, R. (2007). Amygdala damage impairs emotion recognition from music. Neuropsychologia, 45, 236-244.

Hardy, J., Hall, C., \& Alexander, M.R. (2001). Exploring self-talk and affective states in sport. Journal of Sports Sciences, 19, 469-475.

Jones, M.V. (2003). Controlling emotions in sport. The Sport Psychologist, 17, 471-486.

Kallinen, K., \& Ravaja, N. (2004). The role of personality in emotional responses to music: Verbal, electrocortical and cardiovascular measures. Journal of New Music Research, 33, 399-409.

Lang, P.J. (2000). Emotion and motivation: attention, perception, and action. Journal of Sport \& Exercise Psychology, 22(2, Suppl) S122-s140.

Larsen, R.J., \& Diener, E. (1992). Promises and problems with the circumplex model of emotion. In M.S. Clark (Ed.), Emotion (pp. 25-59). Newbury Park, CA: Sage.

Lazarus, R.S., \& Folkman, S. (1984). Stress, appraisal, and coping. New York: SpringerVerlag.

Markman, A.B., \& Brendl, C.M. (2005). Constraining theories of embodied emotion. Psychological Science, 16, 6-10.

Menon, V., \& Levitin, D.J. (2005). The rewards of music listening: Response and physiological connectivity of the mesolimbic system. NeuroImage, 28, 175-184.

North, A.C., \& Hargreaves, D.J. (1995). Subjective complexity, familiarity, and liking for popular music. Psychomusicology, 14, 77-93.

North, A.C., \& Hargreaves, D.J. (1997). Liking, arousal potential, and the emotions expressed by music. Scandinavian Journal of Psychology, 38, 45-53.

Nyklícek, I., Thayer, J.F., \& Van Doornen, L.J.P. (1997). Cardiorespiratory differentiation of musically-induced emotions. Journal of Psychophysiology, 11, 304-321.

Panksepp, J. (1998). Affective neuroscience: The foundations of human and animal emotions. New York: Oxford University Press.

Rickard, N.S. (2004). Intense emotional responses to music: A test of the physiological arousal hypothesis. Psychology of Music, 32, 371-388.

Ritossa, D.A., \& Rickard, N.S. (2004). The relative utility of 'pleasantness' and 'liking' dimensions in predicting the emotions expressed by music. Psychology of Music, 32, $5-22$.

Russell, J.A. (1980). A circumplex model of affect. Journal of Personality and Social Psychology, 39, 1161-1178.

Russell, J.A., Weiss, A., \& Mendelsohn, G.A. (1989). Affect grid: A single-item scale of pleasure and arousal. Journal of Personality and Social Psychology, 57, 493-502.

Scherer, K.R. (1999). Appraisal theory. In T. Dalgleish \& M.J. Power (Eds.), Handbook of cognition and emotion (pp. 637-663). New York: Wiley.

Scherer, K.R. (2004). Which emotions can be induced by music? What are the underlying mechanisms? And how can we measure them? Journal of New Music Research, 33, 239-251. 


\section{TSP GALLEY PROOF}

Scherer, K.R., \& Zentner, M.R. (2001). Emotional effects of music: Production rules. In P. Juslin \& J.A. Sloboda (Eds.), Music and emotion: Theory and research (pp. 361-392). Oxford, UK: Oxford University Press.

Schmidt, L.A., \& Trainor, L.J. (2001). Frontal brain electrical activity (EEG) distinguishes valence and intensity of musical emotions. Cognition and Emotion, 15, 487-500.

Tabachnick, B.G., \& Fidell, L.S. (2007). Using multivariate statistics (5th ed.). Needham Heights, MA: Allyn and Bacon.

Tenenbaum, G., \& Elran, E. (2003). Congruence between actual and retrospective reports of emotions for pre- and postcompetition states. Journal of Sport \& Exercise Psychology, 25, 323-340.

Terry, P.C. (2004). Mood and emotions in sport. In T. Morris \& J. Summers (Eds.), Sport psychology: Theory, applications and issues (2nd ed., pp. 48-73). John Wiley \& Sons Australia.

Thayer, J.F., \& Faith, M. (2001). A dynamical systems interpretation of a dimensional model of emotion. Scandinavian Journal of Psychology, 42, 121-133.

Thayer, R.E. (1989). The biopsychology of mood and arousal. Oxford, UK: Oxford University Press.

Tod, D., Iredale, F., \& Gill, N. (2003). 'Psyching-up' and muscular force production. Sports Medicine, 33, 47-58. Tod, D., Iredale, F., \& Gill, N. (2003).

Turner, M.L., Fernandez, J.E., \& Nelson, K. (1996). The effect of music amplitude on the reaction to unexpected visual events. The Journal of General Psychology, 123, $51-62$.

Webster, G.D., \& Weir, C.G. (2005). Emotional responses to music: Interactive effects of mode, texture, and tempo. Motivation and Emotion, 29, 19-39.

Winterer, G., Adams, C.M., Jones, D.W., \& Knutson, B. (2002). Volition to action - An event-related fMRI study. NeuroImage, 17, 851-858.

Wright, M.J., \& Jackson, R.C. (2007). Brain regions concerned with perceptual skills in tennis: An fMRI study. International Journal of Psychophysiology, 63, 214-220.

Zentner, M.R., \& Kagan, J. (1996). Perception of music by infants. Nature, 383, 29. 
Author: Read proofs carefully. This is your only opportunity to make changes. No further alterations will be allowed after this point. 